\title{
Creatinine inhibits bacterial replication
}

\author{
Thomas McDonald ${ }^{1}$, Kristen M Drescher ${ }^{2}$, Annika Weber ${ }^{1}$ and Steven Tracy ${ }^{1}$ \\ The Journal of Antibiotics (2012) 65, 153-156; doi:10.1038/ja.2011.131; published online 1 February 2012
}

Keywords: antibacterial; antibiotic; bacteria; creatinine

Widespread antibiotic use has resulted in increased frequency of clinically important bacteria acquiring single or multiple antibiotic resistance. ${ }^{1,2}$ Even antibiotic therapies for relatively trivial afflictions, such as acne, ${ }^{3}$ have promoted development of microbial antibiotic resistance. The availability of non-prescription antibiotics in some areas has also resulted in improper and/or irrational self-medication, further exacerbating this problem. ${ }^{4,5} \mathrm{New}$ antibacterial agents with broad-spectrum impact against both Gram-positive ${ }^{6}$ and Gram-negative ${ }^{7}$ species, as well as against drug-resistant strains such as methicillinresistant Staphylococcus aureus ${ }^{8}$ are needed for wound care and to treat topical and dermatological infections. ${ }^{9}$ A chance observation in our laboratory revealed that creatinine $(\mathrm{CRN}$; creatinine hydrochloride, $\mathrm{CRN}-\mathrm{HCl}$ ) halted the growth of bacteria on nutrient agar plates. CRN is the naturally occurring breakdown product of creatine phosphate, a high-energy molecule used to store and then donate, a high-energy phosphate to ADP for the synthesis of ATP in metabolism. Occurring normally in human blood at concentrations ranging approximately between $50-100 \mu \mathrm{M}$ and in urine at slightly higher levels, CRN is accepted to be a naturally produced inert waste product with no active function, ${ }^{10}$ although a recently published study has challenged this dogma. ${ }^{11}$ We characterized the ability of $\mathrm{CRN}-\mathrm{HCl}$ to inhibit the growth of a wide array of bacterial species, including methicillinresistant Staphylococcus aureus.

CRN-HCl (Sigma-Aldrich; St Louis, MO, USA; F.W.=149.6) was evaluated for its ability to inhibit the growth of different bacteria. Bacterial cultures were propagated in LBG (LB broth, Miller; Fisher Scientific; Fair Lawn, NJ, USA) supplemented with $1 \% \mathrm{w} / \mathrm{v}$ glucose) either in liquid or agar (BD Bacto Agar; Becton Dickinson, Sparks, MD, USA) format. Overnight cultures of Escherichia coli and $S$. aureus were diluted in fresh LBG to $1-5 \times 10^{5}$ colony-forming units (c.f.u.) per $\mathrm{ml}$, aliquoted to $2 \mathrm{ml}$ tube cultures, to each of which was added $\mathrm{CRN}-\mathrm{HCl}$ in increasing $5 \mathrm{~mm}$ increments from $0-50 \mathrm{~mm}$. After shaking at 250 r.p.m. at $37^{\circ} \mathrm{C}$ for $18 \mathrm{~h}$, the $\mathrm{OD}$ at $600 \mathrm{~nm}$ was recorded. E. coli was inhibited from growth in $40 \mathrm{~mm}\left(5.98 \mathrm{mg} \mathrm{m}^{-1}\right) \mathrm{CRN}-\mathrm{HCl}$, while $S$. aureus growth was inhibited at a concentration of $15 \mathrm{~mm}$ (2.24 $\mathrm{mg} \mathrm{ml}^{-1}$ ) CRN-HCl (Figure 1a). These values were taken as a measurement of the MIC for $\mathrm{CRN}-\mathrm{HCl}$ under these specific assay conditions. A similar experiment was performed using a 96-well micro- titer plate format in which triplicate wells were filled with 100ul total volume of $E$. coli or $S$. aureus (final concentration of 1$5 \times 10^{5}$ c.f.u. $\mathrm{ml}^{-1}$ ) in LBG containing increasing concentrations of $\mathrm{CRN}-\mathrm{HCl}$. Following $18 \mathrm{~h}$ stationary incubation at $37^{\circ} \mathrm{C}$, wells were examined visually to ascertain which wells appeared turbid versus clear, then the plate was assayed using an ELISA plate reader (ELX800; Bio-Tek Instruments, Winooski, VT, USA) using a $490 \mathrm{~nm}$ wavelength (Figures $1 \mathrm{~b}$ and $\mathrm{c}$ ). This format confirmed the results from the tube cultures, demonstrating the inhibition of the growth of E. coli and S. aureus at 40 and $15 \mathrm{~mm} \mathrm{CRN}-\mathrm{HCl}$, respectively. Using this microtiter plate format, MIC values for four other bacterial species were established: Brevibacterium linens $(\mathrm{MIC}=10 \mathrm{~mm})$, Bacillus subtilis $(\mathrm{MIC}=20 \mathrm{~mm})$, Pseudomonas aeruginosa $(20 \mathrm{~mm})$ and Streptococcus pyogenes $(\mathrm{MIC}=20 \mathrm{~mm}$; data not shown).

Drug-resistant bacterial strains and other bacterial species were assayed for sensitivity to inhibition of replication by $\mathrm{CRN}-\mathrm{HCl}$ using a disc diffusion assay. Assays were performed as described ${ }^{12}$ with the following modifications. Twenty-five microliters of $2 \mathrm{M} \mathrm{CRN-HCl}$ in water was added to $30 \mathrm{mg}$ of a powdered carrier (Eridex; Cargill Inc., Cedar Rapids, IA, USA) and stirred into a thickened slurry in order to apply the maximum amount of $\mathrm{CRN}-\mathrm{HCl}$ on the disc. Fifty microliters of the slurry containing $5 \mathrm{mg}$ CRN-HCl were applied to $6 \mathrm{~mm}$ diameter sterile dry paper discs (Whatman no. 3 filter paper; Whatman, Piscataway, NJ, USA) that were then inverted onto LBG agar plates containing test bacteria. The LBG agar plates were prepared $15 \mathrm{~min}$ before use by spreading the test bacteria (diluted in phosphate-buffered saline to $1-5 \times 10^{5}$ c.f.u. $\mathrm{ml}^{-1}$ from overnight cultures) to the plate with a sterile cotton swab. The plates with discs were incubated for $15 \mathrm{~h}$ at the temperature appropriate for the particular bacterial species. Clear zones around the discs, indicative of growth inhibition, were measured. As a point of comparison, gentamicin-impregnated discs (GM-10; Becton Dickinson) were tested. For all bacteria but drug-resistant and anaerobic bacteria, for which assays were repeated twice, discs were tested in triplicate on two different days and zones of inhibition were recorded as the average of these six measurements. Variation was $\leqslant 2 \mathrm{~mm}$. Results for the diverse bacterial species assayed using this approach are shown in Table 1. All bacteria tested in this manner, including drug-resistant strains,

${ }^{1}$ Department of Pathology and Microbiology, University of Nebraska Medical Center, 986495 Nebraska Medical Center, Omaha, NE, USA and ${ }^{2}$ Department of Medical Microbiology and Immunology, Creighton University, Medical Center, Omaha, NE, USA

Correspondence: Dr T McDonald, Department of Pathology and Microbiology, University of Nebraska Medical Center, 986495 Nebraska Medical Center, Omaha, NE 68198, USA. E-mail: tmcdonal@unmc.edu

Received 24 August 2011; revised 31 October 2011; accepted 7 December 2011; published online 1 February 2012 

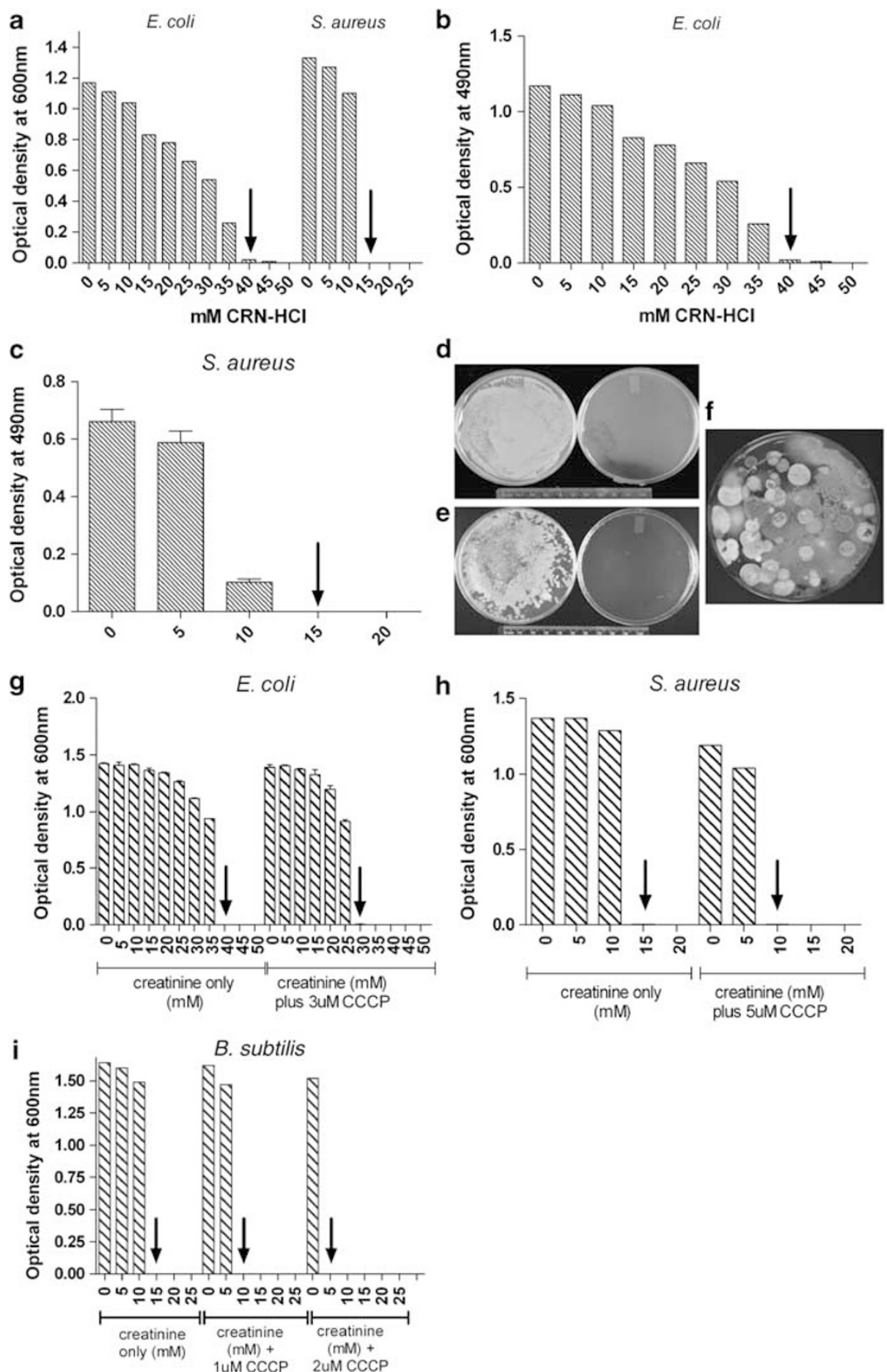

Figure 1 Creatinine inhibits bacterial replication (a-f) potentially by overwhelming the bacterial cells ability to pump out protons (d-i). MIC measurements for CRN-HCl against $E$. coli and $S$. aureus determined in shaking tube culture (a) or stationary 96-well microtiter dish formats $(E$. coli (b); $S$. aureus (c)). The MICs for the two bacterial species were the same regardless of the format employed. Arrows indicate MIC as well as the first tube or well in the series that was not visibly turbid. Replication of bacteria derived from samples of (d) human feces or (e) garden soil was suppressed in the presence of $100 \mathrm{~mm} \mathrm{CRN-HCl}$. Left hand plate in each panel contains no $\mathrm{CRN}-\mathrm{HCl}$; right hand plates contain $100 \mathrm{~mm} \mathrm{CRN-HCl}$. Example of fungal growth following 10 days of incubation at room temperature on an LBG agar plate containing $100 \mathrm{~mm} C R N-\mathrm{HCl}$ (c). The synergy between the inhibitory action of $\mathrm{CRN}-\mathrm{HCl}$ and the action of the proton efflux inhibitor CCCP in lowering the concentration of $\mathrm{CRN}-\mathrm{HCl}$ necessary for growth inhibition is demonstrated for $E$. coli (g), S. aureus (h) and B. subtilis (i). In the presence of CCCP, the MICs are left shifted. Arrows indicate the MIC at which neither detectable turbidity was observed by the eye nor OD when measured at $600 \mathrm{~nm}$. 


\begin{tabular}{|c|c|c|c|}
\hline & \multirow[b]{2}{*}{ Class } & \multicolumn{2}{|c|}{ Zone of inhibition ( $\mathrm{mm}$ ) } \\
\hline & & Creatinine & Gentamicin \\
\hline \multicolumn{4}{|l|}{ Gram-positive bacteria } \\
\hline Staphylococcus aureus & Lab. strain 29213 & 26 & 24 \\
\hline Staphylococcus aureus & UAMS-1 & 24 & ND \\
\hline Staphylococcus aureus & Methicillin resistant (MRSA) & 27 & 7 \\
\hline Staphylococcus epidermidis & Lab. Strain & 27 & ND \\
\hline Enterococcus faecalis & Lab. strain 29212 & 19 & 14 \\
\hline Enterococcus faecium & Vancomycin resistant (VRE) & 18 & 8 \\
\hline Micrococcus luteus & Lab. isolate & 22 & ND \\
\hline Brevibacterium linens & ATCC 9175 & 40 & ND \\
\hline Bacillus subtilis & Lab. strain & 20 & ND \\
\hline Bacillus cereus & Lab. strain & 22 & ND \\
\hline Clostridium difficile & Clinical isolate UNMC 012-SM & 23 & ND \\
\hline Clostridium difficile & Clinical isolate UNMC 013-NO & 21 & ND \\
\hline \multicolumn{4}{|l|}{ Gram-negative bacteria } \\
\hline Pseudomonas aeruginosa & Lab. strain 27853 & 27 & 21 \\
\hline Pseudomonas aeruginosa & HLR & 25 & 8 \\
\hline Pseudomonas fluorescens & Lab. strain & 18 & ND \\
\hline Escherichia coli & Lab. strain 35150 & 21 & 15 \\
\hline Escherichia coli & Beta lactamase producer (ESBL) & 24 & 19 \\
\hline Acinetobacter baumannii & HLR & 16 & 6 \\
\hline Acetobacter xylinum & Lab. strain & 15 & ND \\
\hline \multicolumn{4}{|l|}{ Eukaryotes } \\
\hline Candida albicans & Lab. strain 24433 & 0 & 8 \\
\hline Rhodotorula sp. & Lab. isolate & 0 & ND \\
\hline Saccharomyces & Lab. isolate & 0 & ND \\
\hline
\end{tabular}

Abbreviations: ATCC, American Type Culture Collection; ESBL, extended spectrum beta lactamases; HLR, high-level resistance; Lab. strain, laboratory isolate; MRSA, methicillin-resistant Staphylococcus aureus; ND, Not done; sp., species; UAMS, University of Alabama Medical School; UNMC, University of Nebraska Medical Center; VRE, vancomycin-resistant Enterococcus. Staphylococcus aureus; ND, Not done; sp., species; UAMS, University of Alabama Medical School; UNMC, University of Nebraska Medical Center; VRE, vancomycin-resistant Enterococcus.
Zones of inhibition were determined using disc diffusion assays as described in text. The use of all organisms and procedures were approved by the Institutional Biosafety Committee (IBC) of the Zones of inhibition were determined using disc diffusion assays as described in text. The use of all organisms and procedures We
UNMC, Omaha, NE, USA. Bacterial and yeast species were obtained from Department of Pathology and Microbiology at UNMC.

were inhibited by $\mathrm{CRN}-\mathrm{HCl}$ with a range of zone sizes between $16-40 \mathrm{~mm}$

The ability of CRN-HCl to suppress unknown bacterial growth in environmental samples (fecal matter and soil) was also tested (Figures $1 \mathrm{~d}$ and e). Solids from a human fecal swab were suspended in LBG $(\sim 1 \% \mathrm{w} / \mathrm{v})$ and spread either on control plates containing LBG agar or test plates containing LBG agar poured in $100 \mathrm{~mm}$ CRN$\mathrm{HCl}$. After $24 \mathrm{~h}$ incubation at $37^{\circ} \mathrm{C}$, rampant bacterial growth was observed on the control plate but growth was completely suppressed by $\mathrm{CRN}-\mathrm{HCl}$ (a typical example is shown in Figure 1d; compare control plate on the left to the CRN-HCl-containing plate on the right). Similar experiments using garden soil $(1 \% \mathrm{w} / \mathrm{v})$ as the source of microorganisms gave the same result: complete ablation of bacterial outgrowth in the presence of $\mathrm{CRN}-\mathrm{HCl}$ (Figure 1e). Sterile swabbing of the surface of the clear $\mathrm{CRN}-\mathrm{HCl}$-containing plates (3 days after initial plating) followed by transfer of the swabbed material to control LBG nutrient agar plates, revealed outgrowth of bacterial colonies at varying numbers depending upon the sample used (data not shown). However, none of these outgrowing bacteria replicated when subsequently streaked onto $100 \mathrm{~mm}$ CRN-HCl-containing LBG nutrient agar (data not shown). We infer that these represented outgrowth of persistent bacterial spores that had not previously germinated. Occasionally, growth of diverse fungi on $\mathrm{CRN}-\mathrm{HCl}$-containing agar days after the plating environmental samples was observed, indicating that eukaryotic microorganisms were not inhibited by $\mathrm{CRN}-\mathrm{HCl}$ (Figure 1e). We therefore tested the ability of $100 \mathrm{~mm} \mathrm{CRN}-\mathrm{HCl}$ to inhibit the growth of three different yeasts (Candida albicans, a Rhodotorula species, and a Saccharomyces species; Table 1). No growth inhibition of any of the yeast strains was observed on these plates (similar results were obtained in liquid culture containing 100$500 \mathrm{~mm}$ CRN-HCl; data not shown).

Preliminary studies revealed that $\mathrm{CRN}-\mathrm{HCl}$ - but neither the nonsalt anhydrous form of CRN (Sigma-Aldrich) nor the creatine monohydrate (Creapure; Degussa AG, Dusseldorf, Germany) inhibited bacterial growth (data not shown). Using anhydrous CRN, we formed acetate and sulfate salts of CRN and showed that these, too, functioned as antibacterial agents similar to $\mathrm{CRN}-\mathrm{HCl}$ (data not shown). We hypothesized therefore that only the protonated form $^{13}$ of CRN inhibited the growth of diverse bacterial species, perhaps by overwhelming the bacterial cells capacity to pump out protons. ${ }^{14,15}$ To test this hypothesis, we used $\mathrm{CCCP}^{14}$ (carbonyl cyanide 3-chlorophenylhydrazone; Sigma-Aldrich) to disrupt the proton gradient in cultures of E. coli, S. aureus, and B. subtilis. Tube cultures from diluted overnight cultures were established as described above, each containing the concentration of CCCP and CRN-HCl noted in the figure. Following $18 \mathrm{~h}$ shaking at 250 r.p.m. at $37^{\circ} \mathrm{C}$, the 
OD for each culture was read at $600 \mathrm{~nm}$. Each bacterial species required a 5-10 mm lower $\mathrm{CRN}-\mathrm{HCl}$ concentration to inhibit replication in the presence of $1-5 \mu \mathrm{M}$ CCCP as measured by OD of overnight cultures (Figures $1 \mathrm{~g}, \mathrm{~h}$ and i).

It is widely accepted that CRN is a pharmacologically inactive waste product of muscle metabolism devoid of biological activity. ${ }^{10,16}$ Thus, our discovery that this common nitrogenous waste molecule has antibacterial antibiotic properties is, to the best of our knowledge, unique and potentially of great interest. When tested at physiological levels $(50-200 \mu \mathrm{M})$ on agar or in broth culture, CRN-HCl did not suppress bacterial growth relative to control cultures (data not shown) but when used between 10-100 mM, suppression of growth and killing of all bacterial species were observed including not only drug-resistant strains, such as methicillin-resistant Staphylococcus aureus, but also uncharacterized environmental bacterial strains as well. The mechanism of action is as yet unclear. We know that Streptococcus species, which lack the citric acid cycle ${ }^{17}$ and Staphylococcus species, which use the citric acid pathway, were both inhibited by $\mathrm{CRN}-\mathrm{HCl}$ (data not shown). $\mathrm{CRN}-\mathrm{HCl}$ also inhibited the growth of an arginine-deficient mutant of Staphylococcus epidermidis compared with the wild-type control strain of S. epidermidis (both courtesy of Dr P Fey), indicating the CRN-HCl-induced growth inhibition was not arginine deaminase dependent. ${ }^{18}$ As Clostridium difficile growth and replication was inhibited under anaerobic conditions, thus, we can infer that a lack of oxygen does not affect the suppressive mechanism of CRN-HCl. Our observations that CRN required protonation for the antibacterial function and that inhibition of proton conductance by CCCP lowered the concentration of $\mathrm{CRN}-\mathrm{HCl}$ required to suppress bacterial growth are consistent with a working hypothesis that inhibitory $\mathrm{CRN}-\mathrm{HCl}$ concentrations lead to acidification of the bacterial cytoplasm. Collectively, the results indicate that the mechanism of the suppressive action of $\mathrm{CRN}-\mathrm{HCl}$ used in this study is likely held in common across diverse bacterial species.

The antibacterial characteristics suggest CRN-HCl or other salts of CRN may have potential for the addition to the armamentarium of agents to inhibit dermatological bacterial infections. We propose that $\mathrm{CRN}-\mathrm{HCl}$ may find diverse applications in wound care and treatment as a supplement to, or replacement for, currently used antibacterial agents. Various other clinical, veterinary and industrial applications of CRN-HCL can also be envisaged.

\section{ACKNOWLEDGEMENTS}

We thank Drs Peter Iwen and Paul Fey (University of Nebraska Medical Center, Omaha NE) for their advice and gracious assistance in assaying restricted bacterial species, and for their useful discussions and for providing various S. aureus strains. This study was funded entirely by departmental funds made available to TM and ST.

1 Andersson, D. \& Hughes, D. Antibiotic resistance and its cost: is it possible to reverse resistance? Nat. Rev. Microbiol. 8, 260-271 (2010).

2 Boucher, $\mathrm{H}$. et al. Bad bugs, no drugs: no ESKAPE! An update from the Infectious Diseases Society of America. Clin. Infect. Dis. 48, 1-12 (2009).

3 Patel, M., Boew, W., Heughebaert, C. \& Shalita, A. R. The development of antimicrobial resistance due to antibiotic treatment of acne vulgaris: a review. J. Drugs Dermatol. 9, 655-664 (2010).

4 Vannanen, M., Pietila, K. \& Airaksanen, M. Self-medication with antibiotics - does it really happen in Europe? Health Policy 77, 166-171 (2006).

5 Grigoryan, J. et al. Is self-medication with antibiotics in Europe driven by prescribed use? J. Antimicrob. Chemother. 59, 152-156 (2007).

6 Cornaglia, G. \& Rossolini, G. Forthcoming therapeutic perspectives for infections due to multidrug resistant Gram-positive pathogens. Clin. Microbiol. Infect. 15, 281-323 (2009).

7 Peleg, A. \& Hooper, D. Hospital-acquired infections due to gram negative bacteria. New Engl. J. Med. 362, 1804-1813 (2010).

8 David, M. Z. \& Daum, R. S. Community-associated methicillin-resistant Staphylococcus aureus: epidemiological and clinical consequences of an emerging epidemic. Clin. Microbiol. Rev. 23, 616-687 (2010).

9 Lipsky, B. \& Hoey, C. Topical antimicrobial therapy for treating chronic wounds. Clin. Practice 49, 1541-1549 (2009).

10 Harris,, R. \& Crabb,, D. in Textbook of Biochemistry with Clinical Correlations (ed. Devlin, T.) 884 (Wiley-Liss, New York, 2005).

11 Leland, K., Drescher, K. M. \& McDonald, T. L. Effect of creatine, creatinine, and creatine ethyl ester on TLR expression in macrophages. Int/. Immunopharmacol. 11, 1341-1347 (2011).

12 Clinical and Laboratory Standards Institute. Performance Standards for Antimicrobial Disk Susceptibility Tests Clinical and Laboratory Standards Institute (CLSI) document M2-A9 (CLSI, Wayne, PA, 2006).

13 Srinivasan, R. \& Stewart, R. The catalysis of proton exchange in creatinine by general acids and bases. Can. J. Chem. 53, 224-231 (1974).

14 Nikaido, H. \& Takatsuka, Y. Mechanisms of RND multidrug efflux pumps. BBA Proteins Proteomics 1794, 769-781 (2009).

15 Piddock, L. J. Multi-drug resistance efflux pumps - not just for resistance. Nat. Rev. Microbiol. 4, 629-636 (2006).

16 Wyss, M. \& Kaddurah-Daouk, R. Creatine and creatinine metabolism. Phyisol. Rev. 80, 1107-1213 (2000).

17 Somerville, G. et al. Correlation of acetate catabolism and growth yield in Staphylococcus aureus: implications for host-pathogen interactions. Infect. Immun. 71, 4724-4732 (2003).

$18 \mathrm{Zhu}, \mathrm{Y}$. et al. Staphylococcus aureus biofilm metabolism and the influence of arginine on polysaccharide intercellular adhesin synthesis, biofilm formation and pathogenesis. Infect. Immun. 75, 4219-4226 (2007). 